\title{
Radiative Polarization in the BATES South Hall Ring
}

\author{
M.Korostelev and Yu.M.Shatunov \\ Budker Institute of Nuclear Physics, Novosibirsk, 630090, Russia
}

\begin{abstract}
This report describes some aspects of spin matching conditions at storage ring with Siberian snake to reach larger equilibrium polarization degree. An enhancement of the so-called kinetic polarizing mechanism by special polarizing wigglers is considered.
\end{abstract}

\section{INTRODUCTION}

It is good known that the spin dependence of the synchrotron radiation leads to polarization of an electron beam with a characteristic time $\tau$ to the equilibrium value $P_{e q}[1]:$

$$
P_{e q}=-\frac{8}{5 \sqrt{3}} \frac{\alpha_{-}}{\alpha_{+}} ; \quad \tau^{-1}=\frac{5 \sqrt{3}}{8} \frac{e^{2} \hbar \gamma^{5}}{m^{2} c^{3}} \alpha_{+}
$$

where

$$
\begin{aligned}
& \alpha_{-}=\left\langle\frac{\hat{\mathbf{b}}}{|\rho|^{3}}(\hat{\mathbf{n}}-\mathbf{d})\right\rangle \\
& \alpha_{+}=\left\langle\frac{1}{|\rho|^{3}}\left[1-\frac{2}{9}(\hat{\mathbf{n}} \hat{\mathbf{v}})^{2}+\frac{11}{18}|\mathbf{d}|^{2}\right]\right\rangle
\end{aligned}
$$

Here $\hat{\mathbf{n}}$ is a periodical spin solution, $\mathbf{d}=\gamma \frac{\partial \hat{\mathbf{n}}}{\partial \gamma}$ is a spinorbit coupling vector, $\rho$ is bending magnet curvature, $\hat{\mathbf{v}}$ and $\hat{\mathbf{b}}$ are unit vectors in the direction of particle velocity and magnetic field respectively. The average is taken over the ring azimuth $\theta$ and, generally, over beam distribution.

Experimentally this effect have been studied at many machines, when the $\hat{\mathbf{n}}$ vector coincides practically with the guiding field direction. Using one Siberian snake creates an unusual situation, when the direction of the beam polarization is in horizontal plane excepting the snake, and the scalar product of $\hat{\mathbf{b}}$ and $\hat{\mathbf{n}}$ vectors in $\alpha_{-}$expression (2) is zero. However, the equilibrium polarization should differ from zero level due to so-called kinetic polarizing mechanism. Maximum polarization degree about $\sim 80 \%$ may be achieved when $|\mathbf{d}| \approx 1.2$.

\section{SPIN MATCHING CONDITIONS}

In the energy range of few Gev the longitudinally polarized beams can be obtained with the use of Siberian Snake, a special kind of spin rotator, which rotates particle spin by $180^{\circ}$ angle around a direction lying in the horizontal plane. This direction is called the snake axis. An insertion of Siberian snake with longitudinal snake axis provides automatically the longitudinal beam polarization on a ring azimuth opposite to the snake insertion. Such solenoidal snake was developed for BATES SHR [2].

At first-order approximation the $\mathbf{d}$ vector is orthogonal to $\hat{\mathbf{n}}: \mathbf{d}=\operatorname{Re}\left(i D \hat{\eta}^{*}\right)$ where $\hat{\eta}$ is an eigen solution of spin motion equation orthogonal to $\hat{\mathbf{n}}$. As it was shown in the paper [3], in general case the $D$ function can be represented as a sum of two contributions: $D=D_{\gamma}+D_{\beta}$.

$$
\begin{array}{r}
D_{\gamma}=-\frac{\pi}{2} \sin \left(\pi \nu_{0}\right)+i \nu_{0}\left(\pi-\int_{0}^{\theta} K_{z} d \theta^{\prime}\right) \\
D_{\beta}=-\frac{\nu_{0} \pi}{4 \cos \left(\pi \nu_{x}\right)}\left[\cos \left(\pi \nu_{0}\right) \operatorname{Im}\left(e^{\imath \pi \nu_{x}} J(\theta) G_{I x}^{*}\right)+\right. \\
\left.+i \operatorname{Im}\left(e^{\imath \pi \nu_{x}} G_{I z}^{*} J(\theta)\right)\right]
\end{array}
$$

where $G_{I x, z}=f_{I x, z(\text { out })}^{\prime}-f_{I x, z(\text { in })}^{\prime}$ is the difference of the first mode Floquet function derivatives at the entrance of the first solenoid and at the exit from the second one and $J(\theta)=f_{I x} \psi_{x}^{\prime}-f_{I x}^{\prime} \psi_{x}$.

Here $D_{\gamma}$ comes from the direct dependence of the vector $\hat{\mathbf{n}}$ on the particle energy while $D_{\beta}$ results from a jump of betatron amplitudes during an emission of quanta.

A spin matching condition in the case of one Siberian snake is $\left|D_{\beta}\right|=0$, that can be satisfied if $f_{I x(i n)}^{\prime}=$ $f_{I x(\text { out })}^{\prime}$ and $f_{I z(\text { in })}^{\prime}=f_{I z(o u t)}^{\prime}$.

\section{SOUTH HALL RING WITH SIBERIAN SNAKE}

The Fig. 1 shows the location of the Siberian snake in the SHR. For $E=1 \mathrm{GeV}$ the magnetic field of solenoids is $6.586 \mathrm{~T}$. The snake scheme was chosen for the orbital matching. Unfortunaly the proper spin matching with existing snake is not possible, but the contribution of $D_{\beta}$ can be considerably suppressed due to an optics tuning by nearest to Siberian snake pairs of regular machine quadrupole magnets.

After such corrections, the $|\mathbf{d}|$ behavior (solid line) along azimuth of SHR is shown in the Fig. $1 \mathrm{~b}(E=1 \mathrm{GeV})$, together with $D_{\gamma}$ (dashed line) derived from (3).

All calculations here and later were performed with ASPIRRIN code [3]. The Fig. 2 present the dependence of $\tau$ and $P_{e q}$ on the beam energy for the SHR. One can see that the polarization time is too long and the equilibrium polarization degree is small. 


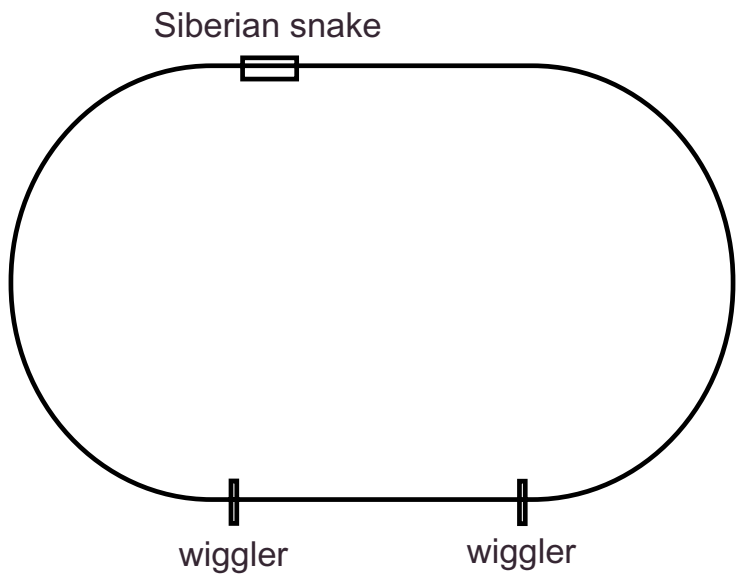

a)

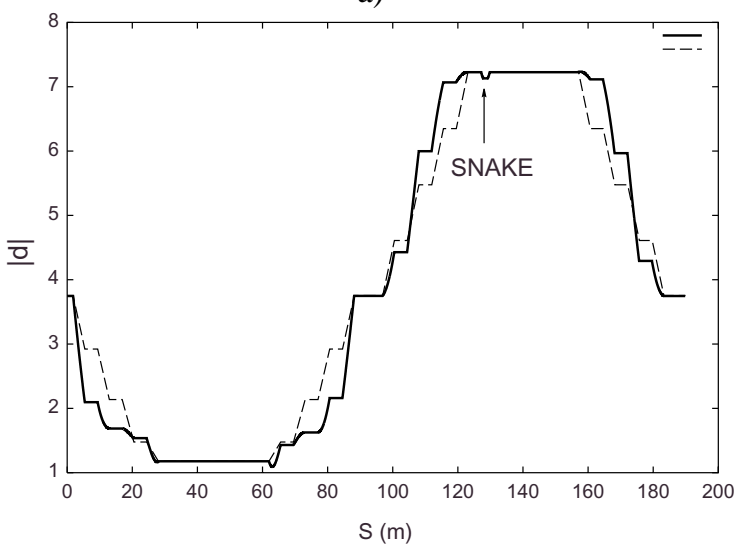

b)

Figure 1: a) Position of Siberian snake in the SHR, b) $|\mathbf{d}|$ along the azimuth of SHR

\section{THE METHOD OF POLARIZATION ENHANCEMENT}

It's good known, that asymmetric wigglers with high magnetic field poles accelerate the radiative polarization. But in the case of machine with Siberian snake an application of the asymmetric wiggler looks much more attractive. If we install such wiggler in the straight section opposite to the snake insertion ( see Fig. 1 ) where $|\mathbf{d}| \approx 1.2$, we get not only faster polarization but also an enhancement of $P_{e q}$.

In this paper we have considered two wigglers with three pairs of magnetic poles. The length of central pole is $10 \mathrm{~cm}$ and its field is $10 \mathrm{~T}$. Both compensated poles are identical. The length and field each of them is $45 \mathrm{~cm}$ and $-1.1111 \mathrm{~T}$ respectively. To compensate the distortion of Floquet functions induced by wiggler, due to strong enough radial focusing, thin quadrupole magnets on both sides of the center pole are needed, namely with $-1.95 \mathrm{KGs} / \mathrm{cm}$ and $10 \mathrm{~cm}$ in length.

The disposition of both wigglers have mirror symmetry with respect to center of straight section. As it is seen from the Fig. 3 the wiggler is located after three quadrupole mag-

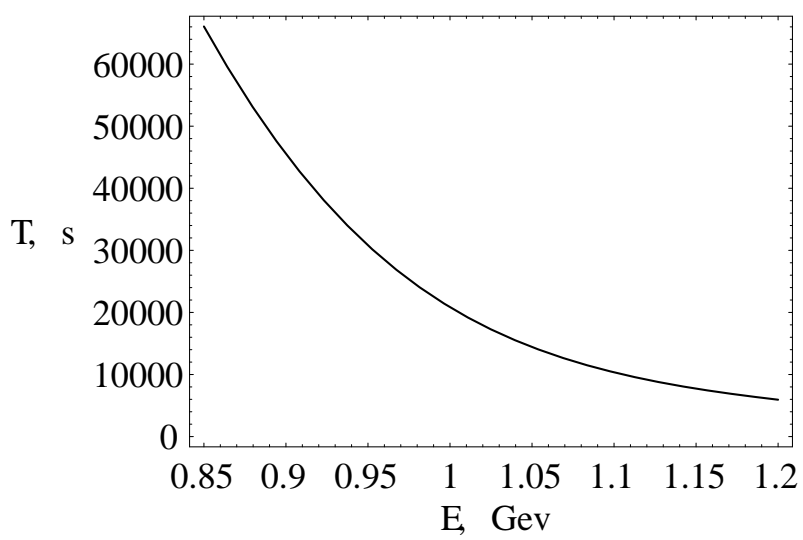

a)

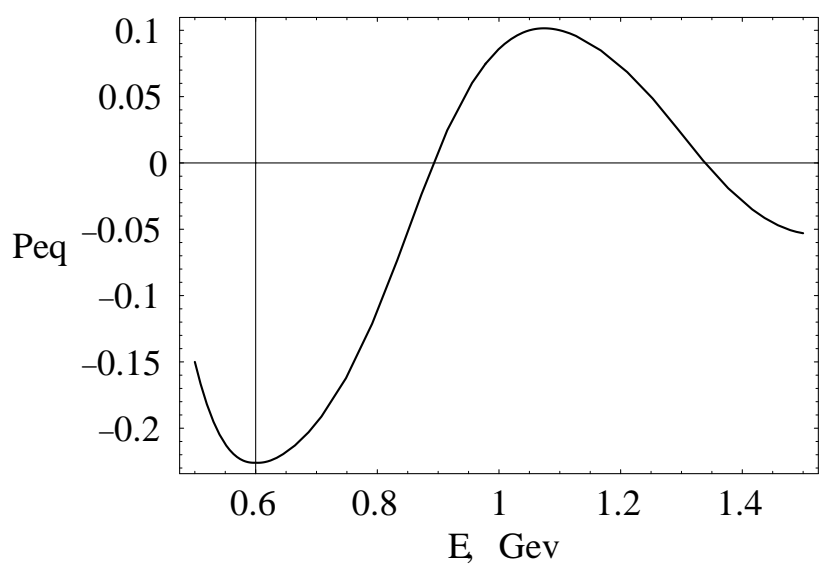

b)

Figure 2: The dependence of polarization time (a) and equilibrium polarization degree (b) on the beam energy.

nets Q6, Q7, Q8 following last arc magnet. Computer simulation shows that we can not restore the original machine optics symmetry by adjustment gradient field of only these (Q6, Q7, Q8) quadrupole magnets. Possible optical solu-

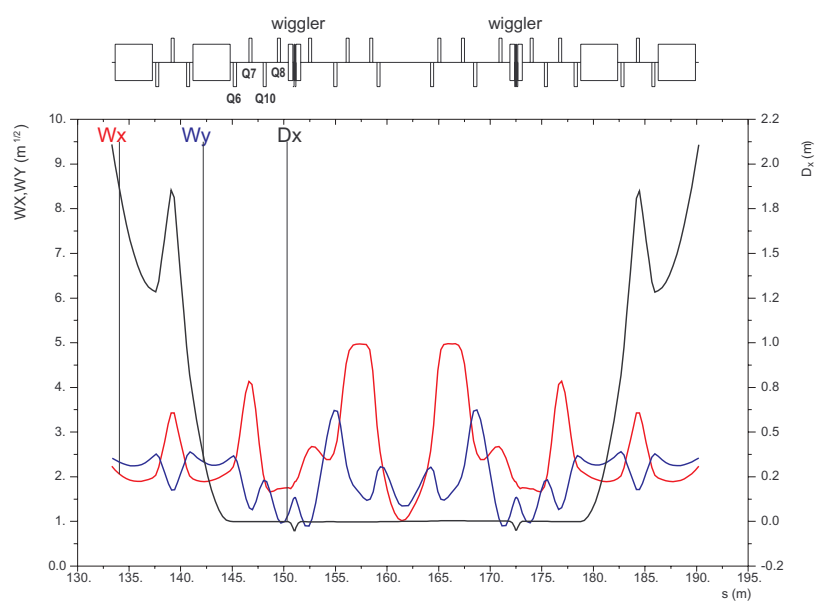

Figure 3: The Floquet and dispersion functions in the straight section with wigglers. 
tion is in a shift of Q8 towards to center of straight section and one additional quadrupole magnet Q10 between Q7 and Q8.

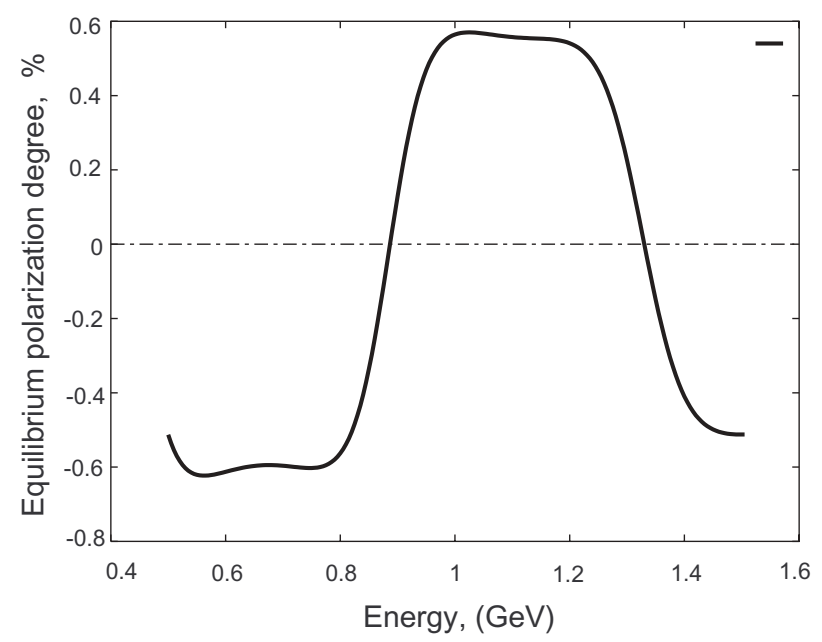

a)

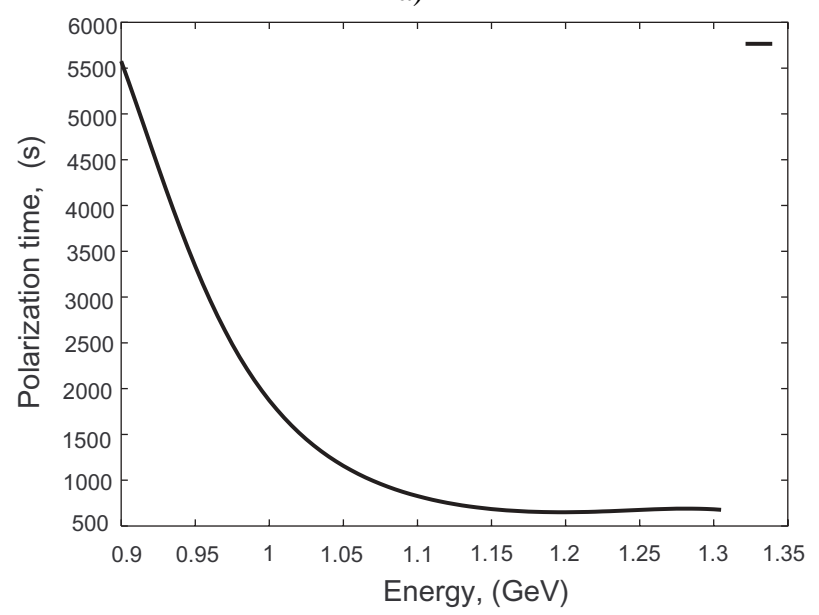

b)

Figure 4: The dependence of polarization time (a) and equilibrium polarization degree (b) on the beam energy with wigglers.
Table 1: The results of the computer simulation with ASPIRIN code for SHR

\begin{tabular}{|l|r|}
\hline Equilibrium polarization degree & $57 \%$ \\
\hline Relaxation time polarization & $1871 \mathrm{~s}$ \\
\hline \multirow{3}{*}{ Betatron tunes } & $\nu_{x}=7.202$ \\
& $\nu_{z}=8.921$ \\
\hline Spin tune & $\nu_{s}=0.5$ \\
\hline
\end{tabular}

The calculation of $P_{e q}$ and $\tau$ for the new lattice with two wigglers are shown in the Fig. 4. The data for the energy $E=1 \mathrm{GeV}$ are presented in the Table 1, from where one can see that we can achieve $57 \%$ polarization degree in spite of discussed above snake imperfections.

Hence the application of the polarizing wigglers can make the kinetic radiative polarization a real instrument for spin experiments at SHR.

\section{REFERENCES}

[1] Ya. S. Derbenev and A. M. Kondratenko, Sov. Phys. JETP, 37, (1973) 968.

[2] S. Kovalsky, T. Zwart, P. Ivanov and Yu. M. Shatunov, Spin Control System for the SHR at Bates linear accelerator center, High Energy Spin Physics eleventh international symposium, Bloomington in 1994.

[3] E. A. Perevedentsev, V. I. Ptitsin and Yu. M. Shatunov, Calculation of spin resonances in storage rings, $\mathrm{V}$ Workshop on High Energy Spin Physics, Protvino, September 1993. 